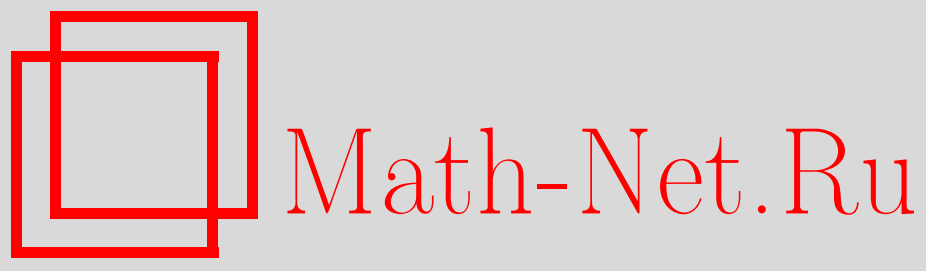

В. М. Мануйлов, К. Томсен, Трансляционно инвариантные асимптотические гомоморфизмы и расширения $C^{*}$-алгебр, Функи. анализ и его прил., 2005, том 39, выпуск 3, 87-91

DOI: https://doi.org/10.4213/faa79

Использование Общероссийского математического портала Math-Net.Ru подразумевает, что вы прочитали и согласны с пользовательским соглашением

http://www . mathnet.ru/rus/agreement

Параметры загрузки:

IP : 54.205 .225 .156

26 апреля 2023 г., 18:23:41

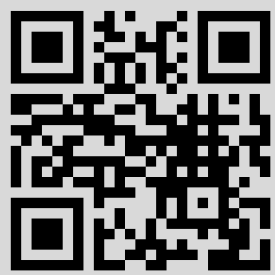




\title{
ЛитерАТУРА
}

1. Бурбаки H. Интегрирование. Меры на локально компактных пространствах. Продолжение меры. Интегрирование мер. Меры на отделимых пространствах. Наука, M., 1977. 2. Gripenberg G., Londen S.-O., Staffans O. Volterra Integral and Functional Equations. Cambridge-New York, Cambridge Univ. Press, 1990. 3. Kolmanovskii V., Myshkis A. Applied Theory of Functional Differential Equations. Dordrecht-BostonLondon, Kluwer Academic Publishers, 1992. 4. Kurbatov V. G. Functional Differential Operators and Equations. Dordrecht-Boston-London, Kluwer Academic Publishers, 1999. 5. Kamenskii G. A., Myshkis A. D. Nonlinear Anal., 34, No. 2, 283-287 (1998). 6. Bлaдимиров B. C. Обобщенные функции в математической физике. Наука, М., 1979. 7. Kurbatov V. G., Studenikin A. A. Funct. Differ. Equ., 4, No. 3-4, 295-327 (1997). 8. Студеникин A. A. Операторы свертки с мерой, сконцентрированной в подполугруппе. Липецк, 1998, Деп. в ВИНИТИ 19.06.98, №1871-B98. 9. Skopin V. A. Funct. Differ. Equ., 7, No. 3-4, 335-371 (2000). 10. Скопин B. A. Дифференциальные уравнения, 37, №9, 1265-1272 (2001). 11. Криштал И. А. Вестник ВГУ, сер. физ. матем., №1, 143-150 (2002). 12. Антоневич А. Б., Лебедев А. В. Труды С.-Петербург. матем. о-ва, 6, 34-140 (1998). 13. Skubachevskii A. L. Elliptic Functional Differential Equations and Applications. Operator Theory, Vol. 91, Basel-Boston-Berlin, Birkhäuser, 1997. 14. Гелъфанд И. М., Райков Д. А., Шилов Г. Е. Коммутативные нормированные кольца. Физматгиз, М., 1960. 15. Бурбаки Н. Спектральная теория. Мир, М., 1972.

Липецкий государственный технический университет e-mail: kv51@inbox.ru, kurbatov@stu.lipetsk.ru

Поступило в редакцию 19 ноября 2003 г.

УДК 517.98

\section{Трансляционно инвариантные асимптотические гомоморфизмы и расширения $C^{*}$-алгебр*}

\author{
(C) 2005. В. М. МАНУЙЛОВ, К. ТОМСЕН
}

Фундаментальная конструкция, введенная в [4] Конном и Хигсоном, сопоставляет расширению сепарабельной $C^{*}$-алгебры $A$ с помощью $\sigma$-унитальной $C^{*}$-алгебры $B$ асимптотический гомоморфизм $S A=C_{0}(\mathbb{R}) \otimes A \rightarrow B$. Выяснение вопроса о том, сколько информации теряется при таком переходе от расширения к построенному по нему асимптотическому гомоморфизму, является трудной задачей. Она особенно интересна в связи с тем, что подход к изучению расширений, развитый Брауном, Дугласом и Филлмором в [3] и обобщенный Каспаровым [7], обнаруживает дефект: в результате не всегда получается групповая структура [2], даже когда гомотопические классы расширений образуют группу [8]. В наших предыдущих работах $[9,11]$ нам удалось в некоторых случаях определить, какая информация содержится в асимптотическом гомоморфизме, полученном конструкцией Конна-Хигсона. Однако в общем случае нам это не удавалось, и результаты настоящей заметки объясняют, почему. Как будет показано, такой асимптотический гомоморфизм обладает свойством, которое до сих пор не было замечено - он трансляционно инвариантен, т. е. сдвигу параметра асимптотического гомоморфизма соответствует такой же сдвиг в $S A$. Мы

* Исследования первого автора частично поддержаны грантом №05-01-00923 РФФИ и грантом НШ-519.2003.1. 
покажем, что эта дополнительная структура асимптотических гомоморфизмов является как раз тем, что нужно для восстановления расширения. Отметим также, что асимптотические гомоморфизмы, построенные в работах [5] и [6] для доказательства теоремы Атьи-Зингера об индексе, являются трансляционно инвариантными.

Пусть $A, B$ суть $C^{*}$-алгебры. Будем говорить, что однопараметрическое семейство $\psi=\left(\psi_{t}\right)_{t \in \mathbb{R}}: A \rightarrow B$ отображений есть асимптотический гомоморфизм (ср. [4]), если

(a) отображение $t \mapsto \psi_{t}(a)$ непрерывно для любого $a \in A$;

(b) $\lim _{t \rightarrow-\infty} \psi_{t}(a)=0$ для любого $a \in A$;

(c) $\lim _{t \rightarrow+\infty}\left\|\psi_{t}(a b)-\psi_{t}(a) \psi_{t}(b)\right\|=\lim _{t \rightarrow+\infty}\left\|\psi_{t}\left(\lambda a+b^{*}\right)-\lambda \psi_{t}(a)-\psi_{t}(b)^{*}\right\|=0$ для любых $a, b \in A, \lambda \in \mathbb{C}$.

Асимптотический гомоморфизм $\psi$ равномерно непреръвен, если таково семейство отображений $\psi_{t}: A \rightarrow B, t \in \mathbb{R}$.

Пусть $\tau=\left(\tau_{s}\right)_{s \in \mathbb{R}}$ - действие группы $\mathbb{R}$ на $S A=C_{0}(\mathbb{R}, A)$ сдвигами, $\tau_{s}(f)(t)$ $=f(t-s), f \in S A$, и пусть $\psi=\left(\psi_{t}\right)_{t \in \mathbb{R}}: S A \rightarrow B-$ асимптотический гомоморфизм. Назовем $\psi$ асимптотически трансляционно инвариантным, если $\lim _{t \rightarrow+\infty}\left(\psi_{t-s}(f)-\psi_{t} \circ \tau_{s}(f)\right)=0$ для всех $s \in \mathbb{R}, f \in S A$, и трансляционно инвариантным, если он равномерно непрерывен и

$$
\psi_{t} \circ \tau_{s}=\psi_{t-s}
$$

для всех $t, s \in \mathbb{R}$. Два (асимптотически) трансляционно инвариантных гомоморфизма $\varphi$ и $\psi$ гомотопны, если существует такой (асимптотически) трансляционно инвариантный гомоморфизм $\Phi: S A \rightarrow I B(=C[0,1] \otimes B)$, что еv 1 ○ $\Phi_{t}=\varphi_{t}$ и $\mathrm{ev}_{0} \circ \Phi_{t}=\psi_{t}$ для всех $t \in \mathbb{R}$, где $\mathrm{ev}_{s}: I B \rightarrow B$ - гомоморфизм вычисления в точке $s \in[0,1]$. Обозначим через $[[S A, B]]_{\tau}$ множество гомотопических классов трансляционно инвариантных гомоморфизмов, а через $[[S A, B]]_{a, \tau}$ множество гомотопических классов асимптотически трансляционно инвариантных гомоморфизмов из $S A$ в $B$.

В дальнейшем будем считать, что алгебра $A$ сепарабельна, а $B \quad \sigma$-унитальна. Обозначим через $M(B)$ алгебру мультипликаторов алгебры $B$, а через $Q(B)$ обобщенную алгебру Калкина $M(B) / B$. Расширение $C^{*}$-алгебры $A$ с помощью $C^{*}$-алгебры $B-$ это короткая точная последовательность $C^{*}$-алгебр $0 \rightarrow B \rightarrow$ $E \rightarrow A \rightarrow 0$. С помощью инварианта Басби такие точные последовательности отождествляются с *-гомоморфизмами из $A$ в $Q(B)$. Пусть $\psi: A \rightarrow Q(B)-$ расширение. Выберем

1) строго положительный элемент $b_{0} \in B, 0 \leqslant b_{0} \leqslant 1$,

2) непрерывное однородное поднятие $s: A \rightarrow M(B)$ расширения $\psi$,

$3)$ последовательность $h_{0} \leqslant h_{1} \leqslant h_{2} \leqslant \cdots$ функций в $C_{0}(0,1]$, удовлетворяющих равенству $h_{n+1} h_{n}=h_{n}$ для всех $n$,

такие, что последовательность элементов $u_{n}=h_{n}\left(b_{0}\right), n=0,1,2, \ldots$, обладает следующими свойствами:

(i) $\lim _{n \rightarrow \infty}\left(u_{n} s(a)-s(a) u_{n}\right)=0$ для всех $a \in A$,

(ii) $\lim _{n \rightarrow \infty} u_{n} b=b$ для всех $b \in B$. 
Это можно сделать тем же способом, которым доказывается существование квазицентральных аппроксимативных единиц [1]. Последовательность $\left\{u_{n}\right\}_{n=0}^{\infty}$ является единичной последовательностъю в смысле работ [9] и [10].

Положим $\Delta_{0}=\sqrt{u_{0}}$ и $\Delta_{n}=\sqrt{u_{n}-u_{n-1}}, n \geqslant 1$. Существенно, что из свойства (i) следует равенство $\Delta_{i} \Delta_{j}=0$ при $|i-j| \geqslant 2$. Пусть $t_{0} \leqslant t_{1} \leqslant t_{2} \leqslant \cdots-$ такая последовательность в $[0,+\infty)$, что $\lim _{n \rightarrow \infty} t_{n}=+\infty$ и $\lim _{n \rightarrow \infty}\left(t_{n}-t_{n+1}\right)=0$.

Легко проверить, что для любой ограниченной по норме последовательности $\left\{m_{j}\right\} \subseteq M(B)$ и для любого $k \in \mathbb{N}$ сумма $\sum_{j=0}^{\infty} \Delta_{j} m_{j} \Delta_{j+k}$ сходится в строгой топологии в $M(B)$ и

$$
\left\|\sum_{j=0}^{\infty} \Delta_{j} m_{j} \Delta_{j+k}\right\| \leqslant \sup _{j}\left\|m_{j}\right\| .
$$

Кроме того, $\sum_{j=0}^{\infty} \Delta_{j} m_{j} \Delta_{j+k} \in B$, если $\lim _{j \rightarrow \infty}\left\|m_{j}\right\|=0$.

Определим асимптотический гомоморфизм $\varphi_{t}: S A \rightarrow B, t \in \mathbb{R}$, формулой

$$
\varphi_{t}(f)=\sum_{j=0}^{\infty} \Delta_{j} s\left(f\left(t-t_{j}\right)\right) \Delta_{j} .
$$

ЛЕмма 1. Асимптотический гомоморбизм $\left(\varphi_{t}\right)_{t \in \mathbb{R}}$ трансляционно инвариaнтен.

ТЕОрема 2. Гомотопический класс трансляционно инвариантного асимптотического гомоморфизма ч не зависит от выбора последовательности $\left\{t_{n}\right\}_{n=0}^{\infty}$, единичной последовательности, строго положстельного элемента $b_{0}$ и поднятия $s$, а зависит лишь от гомотопического класса расширения $\psi$.

Другими словами, если мы обозначаем через $\operatorname{Ext}_{h}(A, B)$ множество гомотопических классов расширений $C^{*}$-алгебры $A$ с помощью $C^{*}$-алгебры $B$, формула (1) определяет отображение

$$
C H_{\tau}: \operatorname{Ext}_{h}(A, B) \rightarrow[[S A, B]]_{\tau} .
$$

В силу сепарабельности $C^{*}$-алгебры $A$ существует компактное множество $X \subseteq A$, линейная оболочка которого плотна в $A$. Заметим, что последовательность $h_{0} \leqslant h_{1} \leqslant h_{2} \leqslant \cdots$ можно выбрать так, чтобы выполнялись условия

$$
\sum_{n=0}^{\infty}\left\|\left[\Delta_{n}, s(a)\right]\right\|<\infty
$$

для всех $a \in X$ и

$$
s(a)-\sum_{j=0}^{\infty} \Delta_{j} s(a) \Delta_{j} \in B
$$

для всех $a \in A$.

Пусть $\kappa: \mathbb{R} \rightarrow(0,1)$ - такая непрерывная возрастающая функция, что $\lim _{t \rightarrow-\infty} \kappa(t)=0$ и $\lim _{t \rightarrow+\infty} \kappa(t)=1$. Положим $v_{t}=\sum_{j=0}^{\infty} \Delta_{j}^{2} \kappa\left(t-t_{j}\right)$.

ЛЕмма 3. Пусть последователъность $h_{0} \leqslant h_{1} \leqslant h_{2} \leqslant \cdots$ выбрана так, что условия (2) и (3) выполнены. Тогда

1) $\lim _{t \rightarrow-\infty} v_{t}=0$,

2) $\lim _{t \rightarrow \infty} v_{t} b=b$ для всех $b \in B$,

3) $\lim _{t \rightarrow \infty}\left(v_{t} s(a)-s(a) v_{t}\right)=0$ для всех $a \in A$,

4) $\lim _{t \rightarrow \infty}\left(g\left(v_{t}\right) s(a)-\varphi_{t}((g \circ \kappa) \otimes a)\right)=0$ для всех $g \in C_{0}(0,1), a \in A$. 
Это значит, что если $C H(\psi): S A \rightarrow B$ - асимптотический гомоморфизм, полученный с помощью конструкции Конна-Хигсона [4], то $C H(\psi)$ гомотопен трансляционно инвариантному асимптотическому гомоморфизму.

Перейдем теперь к построению обратного к $C H_{\tau}$ отображения. Пусть $\beta_{0} \in$ $C_{0}(\mathbb{R})$ - такая непрерывная функция с носителем в $[-1,1]$, что

$$
\beta_{0}(t)>0 \quad \text { при } t \in(-1,1), \quad \tau_{1}\left(\beta_{0}\right) \tau_{-1}\left(\beta_{0}\right)=0
$$

и

$$
\tau_{-1}\left(\beta_{0}\right) \beta_{0}+\beta_{0}^{2}+\tau_{1}\left(\beta_{0}\right) \beta_{0}=\beta_{0} .
$$

Для $i \in \mathbb{Z}, i \neq 0$, положим $\beta_{i}=\tau_{-i}\left(\beta_{0}\right)$. Тогда $\left\{\beta_{i}\right\}_{i \in \mathbb{Z}}-$ такое разбиение единицы в $C_{0}(\mathbb{R})$, что $\operatorname{supp} \beta_{i}=[-i-1,-i+1]$. Положим $\alpha_{i}=\sqrt{\beta_{i}}$ и заметим, что $\alpha_{i} \alpha_{j}=0,|i-j| \geqslant 2$.

Пусть $\varphi=\left(\varphi_{t}\right)_{t \in \mathbb{R}}: S A \rightarrow B-$ асимптотически трансляционно инвариантный асимптотический гомоморфизм. Для удобства предположим, что $\varphi_{t}(0)=0$ при всех $t$. Тогда

$$
\varphi_{i}\left(\tau_{i}\left(\alpha_{i} \alpha_{j}\right) \otimes a\right)=0 \quad \text { при }|i-j| \geqslant 2
$$

для всех $a \in A$. Пусть $\mathbb{K}$ есть $C^{*}$-алгебра компактных операторов в $l^{2}(\mathbb{Z})$, и пусть $e_{i j}, i, j \in \mathbb{Z},-$ стандартные матричные единицы в $\mathbb{K}$.

Определим отображение $\Phi_{0}: A \rightarrow M(B \otimes \mathbb{K})$ равенством

$$
\Phi_{0}(a)=\sum_{i, j \in \mathbb{Z}} \varphi_{i}\left(\tau_{i}\left(\alpha_{i} \alpha_{j}\right) \otimes a\right) \otimes e_{i j} .
$$

Эта сумма сходится в строгой топологии благодаря свойству (4) и оценке $\sup _{i, j}\left\|\varphi_{i}\left(\tau_{i}\left(\alpha_{i} \alpha_{j}\right) \otimes a\right)\right\|<\infty$. Пусть $q_{B \otimes \mathbb{K}}: M(B \otimes \mathbb{K}) \rightarrow Q(B \otimes \mathbb{K})-$ канонический эпиморфизм и $\Phi=q_{B \otimes \mathbb{K}} \circ \Phi_{0}$.

ЛЕмма 4. Отображение $\Phi$ является *-гомоморфизмом из $A$ в $Q(B \otimes \mathbb{K})$, m. е. расширением $C^{*}$-алгебры $A$ с помощъю $C^{*}$-алгебры $B \otimes \mathbb{K}$.

Обозначим через $I(\varphi)$ расширение $\Phi$ из леммы 4 . Легко видеть, что эта конструкция дает корректно определенное отображение

$$
I:[[S A, B]]_{a, \tau} \rightarrow \operatorname{Ext}_{h}(A, B \otimes \mathbb{K}),
$$

такое, что $I[\varphi]=[I(\varphi)]$.

Tеорема 5. Пусть $A$ и $B$ суть $C^{*}$-алгебры, $A$ сепарабелъна, а $B$ б-унитальна и стабильна. Тогда имеет место изоморфизм $[[S A, B]]_{a, \tau} \simeq[[S A, B]]_{\tau}$ (как абелевых полугрупп) и $C H_{\tau}: \operatorname{Ext}_{h}(A, B) \rightarrow[[S A, B]]_{\tau}-$ изоморфизм.

В заключение отметим, что $[[S A, B \otimes \mathbb{K}]]_{\tau}$ совпадает с группой $E(S A, B)$ $E$-теории по крайней мере в двух известных случаях: когда алгебра $A$ ядерна и когда она является надстройкой.

\section{ЛитеРАТУРА}

1. Arveson W. Duke Math. J., 44, 329-355 (1977). 2. Anderson J. Ann. of Math., 107, 455-458 (1978). 3. Brown L. G., Douglas R. G., Fillmore P. A. Ann. of Math., 105, 265-324 (1977). 4. Connes A., Higson N. C. R. Acad. Sci. Paris Sér. I Math., 311, 101-106 (1990). 5. Higson N. In: Index theory and operator algebras (Boulder, CO, 1991), Contemp. Math., Vol. 148, Amer. Math. Soc., Providence, RI, 1993, pp. 67-86. 6. Higson N., Kasparov G., Trout J. Adv. Math., 135, 1-40 (1998). 7. Каспаров Г. Г. Изв. АН СССР, сер. матем., 
44, 571-636 (1980). 8. Kirchberg E. Invent. Math., 112, 449-489 (1993). 9. Manuilov V. M., Thomsen K. J. Funct. Anal., 213, 154-175 (2004). 10. Manuilov V. M., Thomsen K. Proc. London Math. Soc., 88, 455-478 (2004). 11. Manuilov V. M., Thomsen K. K-Theory, 32, 101-138 (2004).

Московский государственный университет, механико-математический факультет,

Поступило в редакцию e-mail: manuilov@mech.math.msu.su 30 января 2004 г.

Institut for Matematiske Fag, Ny Munkegade, Denmark e-mail: matkt@imf.au.dk

УДК 517.43

\title{
Коммутирующие дифференциальные операторы ранга 2, отвечающие кривой рода $2^{*}$
}

\author{
(c) 2005. А. Е. Миронов
}

1. Введение. В данной статье построены некоторые обыкновенные формально самосопряженные коммутирующие дифференциальные операторы ранга 2 , отвечающие кривой рода 2 , причем функция Бейкера-Ахиезера этих операторов имеет существенную особенность в точке Вейерштрасса.

Обозначим через $L_{1}$ и $L_{2}$ дифференциальные операторы

$$
L_{1}=\partial_{x}^{n}+\sum_{i=0}^{n-2} u_{i}(x) \partial_{x}^{i}, \quad L_{2}=\partial_{x}^{m}+\sum_{i=0}^{m-2} v_{i}(x) \partial_{x}^{i} .
$$

Напомним, что по лемме Берчналла-Чаунди, если $L_{1} L_{2}=L_{2} L_{1}$, то существует полином $Q(z, w)$ от двух коммутирующих переменных, такой, что $Q\left(L_{1}, L_{2}\right)=0$. Обозначим через Г кривую, заданную в плоскости $(z, w)$ уравнением $Q(z, w)=0$. Для $P \in \Gamma$ в общем положении существует совместная собственная функция $\psi(x, P)$ (функция Бейкера-Ахиезера) операторов $L_{1}$ и $L_{2}$, такая, что $L_{1} \psi=z \psi$, $L_{2} \psi=w \psi$. Размерность $l$ пространства таких функций, отвечающих $P$, называется рангом пары $L_{1}, L_{2}$. Для операторов ранга 1 функция $\psi$ выражается явным образом через тэта-функцию многообразия Якоби кривой Г, и в этом случае коэффициенты операторов $L_{1}$ и $L_{2}$ легко найти [1]. Случай $l>1$ существенно сложнее. Задача отыскания операторов ранга $l>1$ в общем случае до сих пор не решена. Новиков и Кричевер [2] нашли операторы ранга 2, отвечающие кривой рода 1. Мохов [3] нашел операторы ранга 3 рода 1.

Основной результат этой работы заключается в следующем. Пусть $l=2$ и кривая Г рода 2 является гладким пополнением бесконечно удаленной точкой $\infty$ кривой, заданной в $\mathbb{C}^{2}$ уравнением

$$
w^{2}=F(z)=z^{5}+c_{3} z^{3}+c_{2} z^{2}+c_{1} z+c_{0} .
$$

Кривая $Г$ допускает голоморфную инволюцию $\sigma: \Gamma \rightarrow \Gamma, \sigma(z, w)=(z,-w)$, которая имеет 6 неподвижных точек $\infty,\left(z_{i}, 0\right)$, где $z_{i}$ - точки ветвления (корни уравнения $F(z)=0)$.

*Работа поддержана грантами РФФИ (03-01-00403 и 03-01-06482) и грантом НШ2185.2003 .1 